\title{
KSENIA KUZMINYKH
}

\author{
Georg-August-Universität Göttingen \\ kkuzmin@gwdg.de \\ ORCID: 0000-0003-0744-4010
}

\section{Literalität in kinder- und jugendliterarischen Werken}

\section{Literacy in children and juvenile books}

\begin{abstract}
The article starts with a discussion of the essential theories of literature. It focuses on the historical development of books for children and young-adults. Worldwide there are three childhood myths, which are unfolded in successful children's books and which correspond to socially conditioned concepts of childhood. The Enlightenment childhood utopia sees children as promising for the future and improving human relationships. This idea explains the phenomenal resonance of books with educational and instructive concepts. In the $20^{\text {th }}$ and $21^{\text {st }}$ centuries this concept has become very popular again. By contrast, Romanticism developed another, second childhood myth, which combines not a future but a paradisiacal past with the image of childhood. In doing so, the holistic and naïve childlike world reference is stylized into an ideal that expresses the backward-looking yearning of adults. In addition to the Enlightenment and the Romantic childhood myths, there is a third, a negative view of childhood, which has also found expression in children's classics worldwide. This refers to the myth of the evil child, who is originally committed to the Christian doctrine of original sin. In the next step the article traces different modes of reading. These are the literary mode and documentary/pragmatic mode. Then, based on fragments taken from children's and juvenile books from different periods, the article demonstrates the role of literacy in texts written for children and young adults, and the role of children and young-adult books for reading competence. While the paper examines these ideas within different novels though history, it also raises questions about the aesthetics and epistemic value of literature.
\end{abstract}

KEYWORDS: literature, literacy, literary mode, pragmatic mode, documentary mode, history of children and, young-adult books, motivation to read, the epistemic value of literature.

SCHLÜSSELWORTE: Literatur, Literalität, ästhetische Rezeptionsmodus, pragmatischer Rezeptionsmodus, dokumentarischer Rezeptionsmodus, Kinder- und Jugendliteratur, Lesemotivation, der epistemische Wert der Literatur. 


\section{EINLEITUNG: HYPOTHESEN UND GRUNDLEGENDE ÜBERLEGUNGEN}

Anhand von einigen ausgewählten kinder- und jugendliterarischen Werken soll gezeigt werden, welcher Stellenwert der Literalität und dem literarisch-ästhetischen Genuss in Literatur zukommt, und mit welchen Strategien die kindlichen und jugendlichen Leserinnen und Leser zum Lesen hingeführt werden. Zu Beginn finden sich Überlegungen zur Kinder- und Jugendliteratur. Dann folgt ein kurzer Einblick in ihre historische Entwicklung im europäischen Raum. Dabei werden differierende Vorstellungen von dem, was in die Gruppe der kinderliterarischen Werke eingeordnet werden kann, und Ziele, die man damit verfolgte, fokussiert. Diese Dissonanz erklärt partiell auseinandergehende Vorstellungen von der Literalität und von den Wegen, diese zu erlangen. Weiterhin widmet sich die Aufmerksamkeit der sprachlich-ästhetischen Verfasstheit der Texte, um schließlich zu der Thematisierung des literacy-Phänomens in Kinder- und Jugendliteratur, dem zentralen, eingangs formulierten Anliegen zu kommen.

\section{DISKURSIVE VERHANDLUNGEN VON LITERATUR UND SPRACHE IN KINDER- UND JUGENDLITERATUR}

Das intensive Leseerleben und eine evasorische Rezeptionssituation werden als spezifisch kindliche Leseverhaltensweisen beschrieben. Ein idealer Leser ist dementsprechend ein in ein Buch versinkender, kindlicher Leser:

In anything fit to be called by the name of reading, the process itself should be absorbing and voluptuous; we should gloat over a book, be rapt clean out of ourselves, and rise from the perusal, our mind filled with the busiest, kaleidoscopic dance of images, incapable of sleep or of continuous thought. The words, if the book be eloquent, should be thenceforward in our ears like the noise of breakers, or the story, if it be a story, repeat itself in a thousand coloured pictures to the eye. It was for this last pleasure that we read so closely, and loved our books so dearly, in the bright, troubled period of boyhood (Stevenson 1988: 172).

Die Suche nach einer adäquaten Definition des Phänomens Kinder- und Jugendliteratur erweist sich aufgrund der Inhomogenität des Gegenstandes als schwierig. Die Begriffserklärung kann aus der Perspektive der Rezeptionsorientierung vorgenommen werden. Man kann aber von einem besonderen Modus der Literatur ausgehen - "unique literary mode in that the sender and the receiver of the text are on different cognitive levels" (Nikolajeva 2014: 13). 
Dabei ist dem Autor per difenitionem ein kognitiver und emotionaler Wissensvorsprung inhärent (Stockwell 2002). Allerdings ist bei dieser Begriffserklärung der Akzent auf den Ausdruck per difenitionem zu legen, denn eine Diskrepanz zwischen dem Autor und dem Leser kann immer, also auch in der Literatur für Erwachsene bestehen. Die Begriffserklärung des Phänomens lässt sich aus der Sicht der Einrichtungen, die sich an der Vermittlung von kinder- und jugendliterarischen Werken und somit an der Lesesozialisation beteiligen, vornehmen. Ewers (2012) weist darauf hin, dass Kinder- und Jugendliteratur, das ist, was als Kinderbuch kommuniziert wird, und erst dann auch das, was als Kinderbuch geschrieben ist.

Theodor Storm formulierte die Maxime der dichterischen Form für junge Leserinnen und Leser: „Wenn du für die Jugend schreiben willst, so darfst du nicht für die Jugend schreiben“ (Storm 1877: 107). Damit deutet der Vertreter des Poetischen Realismus auf die Unabhängigkeit der Qualität des literarischen Schreibens vom Alter seines anvisierten Lesepublikums und legt nahe „eine Schreibweise zu entwickeln, die sowohl den reifen Menschen anspreche, als auch die Teilhabe der Jugend [an der hohen Literatur] erwecke" (Storm 1877: 107). Es geht somit stets um die Balance zwischen der Elementarisierung und der Anpassung an die Dispositionen der Leseanfänger und dem künstlerischen Anspruch. Die Simplizität bedingt ästhetische Komprimierung literarischer Komplexität und legt Transparenz und Stringenz der Darstellung, der Figuren, der Handlung und Handlungslinien, des Plots, der Zeitebenen, der Metafiktion, der Intertextualität, der Form und der Makrostruktur, der Narration und des Stils nahe.

Ewers (2012) differenziert faktische, intendierte, erfolgreiche und nicht erfolgreiche, unbeabsichtigte, positiv und negativ sanktionierte, originäre und spezifische Kinder- und Jugendliteratur. Auch änderten sich vom späten Mittelalter bis in die Gegenwart Vorstellungen von den Konzepten, Inhalten, Zielen und Funktionen der Kinder- und Jugendliteratur.

Für das späte Mittelalter und die frühe Neuzeit ist kennzeichnend, dass die Phase vor dem Eintritt in das Erwachsenenalter in sich kaum differenziert war. Darüber hinaus galten als Kinder nicht nur junge Heranwachsende, sondern grundsätzlich alle illiteraten Personen. Ziele der Literalität bezogen sich vorwiegend auf das Lesenlernen (Abc-Bücher, Erstlesebücher und belehrend-unterhaltende Informationsblätter - im XVI-XVII Jahrhundert), auf die religiöse Unterweisung, auf die Vermittlung richtiger Umgangsformen, der politischen Klugheit und der sprachlich-rhetorischen Bildung und schließlich auf die Förderung des Sachwissens mittels belehrender Anschauung, wenngleich die Illustrationskunst nicht unumstritten war. Dies ist für die gesamte europäische Kinder- und Jugendliteratur kennzeichnend. 
Erst in der Zeit der Aufklärung beginnt sich das zielgerichtete Schreiben für Kinder und Jugendliche als eine kulturelle Praxis $\mathrm{zu}$ etablieren. Dabei wird von den Erkenntnissen der Anthropologie der Kindheit, die kognitive Entwicklung und Sensualität der jungen Rezipientinnen und Rezipienten sowie ihr (visuelles) Unterhaltungsbedürfnis fokussiert, ausgegangen. Kinder- und jugendliterarische Werke erfahren eine adressatenspezifische Akkommodation. Es wird allegorisch, an lehrhaften Beispielen und anhand von didaktisch motivierten Illustrationen in einer übergenerationalen Kommunikationssituation mit Anpassung an die Rezeptionserfahrung erzählt. Als ein Exempel par excellence ist die Überarbeitung des Romans von D. Defoe The Life and Strange, Surprising Adventurens of Robinson Croesoe, of York, Mariner: Who lived Eight and Twenty Years, all alone in an un-Inhabited Island on the Coast of AMERICA, near the Mouth of the Great River of OROONOQUE; Having been cast on Shore by Shipwreck, wherein all the Men perished but himself. WITH An Account how he was at last as strangely deliver'd by PYRATES. Written by Himself von J. Campe Robinson der Jüngere (Campe 1779) zu nennen:

Erstlich wolte ich meine jungen Leser auf eine so angenehme Weise unterhalten, als mir möglich wäre; weil ich wußte, daß die Herzen der Kinder sich jedem nüzlichem Unterrichte nicht lieber öfnen, als wenn sie vergnügt sind (Campe 1779: 5).

Moralisierend sind Fabelsammlungen, die bereits im Namen ihre pädagogischen Intentionen offenbaren. Das Lasterhafte wird ausgelacht, die Selbstkorrektur ist die anzustrebende Maxime (z. B. Weiße 1758).

Ferner sind kinderliterarische Werke dieser Zeit von einem Arbeitsethos und vor allem auf Vernunft, Tugendhaftigkeit und Triebkontrolle gegründeten Menschenbild geprägt. Es gilt das Böse im Kind auszutreiben, eine Vorstellung, die auf die christliche Erbsündenlehre zurückzuführen ist. Werke wie das von A. Bürger (1786) Wunderbare Reisen zu Wasser und Lande, Feldzüge und lustige Abenteuer des Freiherrn von Münchhausen, wie er dieselben bei der Flasche im Zirkel seiner Freunde selbst zu erzählen pflegt (1786, erweiterte Ausgabe 1788) sind eine Ausnahme. Sie werden anonym publiziert und erlangen dadurch keinen sakrosankten Wortlaut, d.h. sie können beliebig geändert und an die Zielgruppen angepasst werden. So erscheint 1842 das Werk illustriert als ein Buch für Kleinkinder. Kunst- und lustvoll phantasierendes Spiel mit dem offensichtlich Unwahrscheinlichen, die komisierende Fiktionalisierung durch Verstoß gegen die Gesetze der Logik, der Erfahrung, der Natur - aber alles mit dem Anspruch unbedingter Glaubwürdigkeit und Seriosität und mit einem Zugriff auf die aufklärerischen Diskursregeln (Verstand) vorgetragen -, erklären den Erfolg des Werks, das mit seinen Prätexten - Schelmuffskys kuriose und sehr gefährliche Reisebeschreibung zu Wasser und 
Land (Reuter 1696) und Reiseberichte Verae historiae von Lukian von Samosata (2014) - nicht als ein Werk der Kinderliteratur konzipiert wurde.

Das Viellesen wird allerdings als Lesewut und Vergnügungssucht negativ konnotiert. Gelesen werden soll nicht rauschhaft, affektgeleitet, sondern reflektierend, gründlich, langsam und aufmerksam die moralische Botschaft aufnehmend.

Für die Periode der Romantik ist ein Paradigmenwechsel in Hinblick auf die Aufwertung des (unterhaltenden) Lesens und des Viellesens kennzeichnend. Dieser geht mit einer Polarisierung einher. Auf der einen Seite ist die aufgeklärt-moralisierende Literatur der Vernunftpädagogik und des Nützlichkeitsdenkens, die zwar sukzessive jedoch nie vollständig aufgegeben wird, zu finden. Auf der anderen Seite positioniert sich die an der Tradition des Volkes orientierte Literatur mit utopisch erhöhten, idealisierten, nahezu mystischen Vorstellungen vom zeitlich begrenzten Entwicklungsphänomen Kindheit als einem goldenen Zeitalter mit eigener Dignität und poetischer Daseinsform. Kinder werden zum Vorbild für Erwachsene. Sie erlangen eine absolute Selbststätigkeit im Bildungsprozess. Dem Kind werden Verbundenheit mit einer übernatürlichen, göttlichen bzw. geistigen Welt, unerschöpfliche Kreativität und die "Überlegenheit in den höchsten Dingen“ attestiert (Novalis 1977: 326).

Für die kinder- und jugendliterarischen Werke sind Sprachbilder kennzeichnend, die zwischen Scherz, Spiel und Phantasie, tiefdimensionaler (sinnlich wahrgenommener) Bedeutung und der Sehnsucht nach Wunderbarem und Numinosem oszillieren (Des Knaben Wunderhorn von Arnim \& Brentano 1808, Kinder- und Hausmärchen der Brüder Grimm 1812/1962). Das Stilprinzip der Heterogenität - literarisch artifizieller Sprachgebrauch einerseits, volkstümliche Sprache andererseits - prägt das narrative Fundament und vervollständigt die Unterscheidungsmerkmale zur pädagogischen Literatur der Aufklärung.

Wir wollten durch unsere Sammlung nicht bloß der Geschichte der Poesie einen Dienst erweisen, es war zugleich Absicht, dass die Poesie selbst, die darin lebendig ist, wirke: erfreue, wen sie erfreuen kann, und darum auch, dass ein eigentliches Erziehungsbuch daraus werde (Grimm 1962: 237).

Es entstehen die geltenden rationalen Normen herausfordernde und den Utilitarismus sowie die verlorene Harmonie zwischen Mensch und Natur kritisierende Texte, beispielsweise Struwwelpeter von H. Hoffmann (1844/1917) und ähnliche wie Struwwel-Lene (Seemann 1898) sowie Exemplare der Antikinder- und Jungendliteratur, in denen Hauptfiguren auf eine grausame und brutale Weise verstümmelt werden und sogar sterben Max und Moritz von W. Busch (1865). 1865 erscheint ein herausragendes Beispiel der Non- 
sens-Literatur Alice in Wonderland von L. Carroll. Diese Werke erfahren eine rasante Verbreitung in übersetzter Form.

Innovativ für die Romantik (und bis in die Gegenwart andauernd) sind die kindlichen Helden (Jean Paul 1808/1963) und die Mehrfachadressierung: Dem (erwachsenen) Vorleser wird nicht lediglich die pädagogische Funktion eines Wertvermittlers zugeschrieben, sondern implizit, auf einer anderen Ebene, ein ästhetischer Genuss und Metakommunikation mit dem Text ermöglicht. Hinter der expliziten, "exotischen Bildebene" (Ewers 2012: 61), die die Kinder faszinieren soll, verbirgt sich auf der Sachebene der latente Sinn, der sich in der polyvalenten Parabolik manifestiert. Es handelt sich hierbei jedoch nicht um eine bloße Zweiteilung der Textdimensionen in eine Ebene für unerfahrene und eine Ebene für erfahrene Leser, sondern um eine Fülle von sich gegenseitig überlagernden Bedeutungstechniken. Das Entdecken der Mehrperspektivität und -dimensionalität wird zu einem unabschließbaren transzendierenden literarischen Spiel für Rezipientinnen und Rezipienten.

Im Jahrhundert des Kindes (Key 1978) spielt die Kinder- und Jugendliteratur mit den Grenzen der Altersgruppen und Genres, mit den soziokulturellen und innerpsychischen Faktoren und der Möglichkeit unterschiedlicher Entwicklungsverläufe. Als einige Beispiele dieser Tendenz sind z. B. Pippi Langstrumpf (Lindgren 1949), Pünktchen und Anton (Kästner 1931/1976) zu nennen. Diese Akzente werden im literarischen Realismus intensiviert.

Im 21. Jahrhundert entwickelt Kinder- und Jugendliteratur eine ästhetische Autonomie (Wrobel 2000). Sie offeriert ihren Rezipientinnen und Rezipienten ein reiches Repertoire literarischer Erfahrungen. Wie auch die allgemeine Literatur behandelt sie zwar allusorisch die Existenzialien des Lebens und wartet mit Motivarsenalen, Protagonisten sowie Handlungsmuster auf, dennoch stellt sie kein Abbild der Wirklichkeit dar. Vielmehr kreieren kinder- und jugendliterarische Texte wie auch Texte der allgemeinen Literatur mit Hilfe stilistischer Mittel und Prinzipien der Abstraktion und Symbolisierung eine mimetische Nähe zur Wirklichkeit. Sie entwerfen bei der Übersetzung des Real-Gegebenen subjektiv-illusionäre Wahrheitsskizzen und entziehen sich einer zuverlässigen Verifikation. Sie bleiben polyvalent, tiefendimensional und mehrperspektivisch. Somit erzielt das erschaffene Wirklichkeitsmodell eine bestimmte Wirkung. Diese geht mit einer besonderen Rezeptionshaltung einher, dem ästhetischen bzw. dem literarischen Rezeptionsmodus. Diesem literary mode (Kintsch 1998) ist eine Bereitschaft der Lesenden zur größeren Ambiguitätstoleranz inhärent (Kuzminykh 2018). Den Gegensatz dazu bildet der dokumentarisierende / pragmatische Lesemodus, in dem die Rezipienten dazu neigen, das Gelesene als reliabel wahrzunehmen (Mitterer \& Wintersteiner 2015). 
Die Kindheit wird im Realismus nicht als ein von Gegebenheiten des Alltags weitgehend verschonter Zustand gesehen, sondern sie mischt sich selbstbewusst in die Welt der Erwachsenen ein und durchbricht die ihr zugewiesenen Grenzen der Unmündigkeit. Die jungen Rezipienten werden zur Reflexion und zum kritischen Hinterfragen der eigenen Einstellung sich selbst, dem und den Anderen, der Diversität des gesellschaftlichen Miteinanderseins gegenüber sowie zu den individuellen Bewertungsvorgängen aufgefordert - ein neuer Zugang zu Problemorientierung und ein völlig neuer Umgang damit, z. B. im Werk Rico, Oscar und die Tieferschatten (Steinhöfel 2008). Konstituierend für die Kinder- und Jugendliteratur sind geschichtserzählende Romane, Reise- und Kriminalerzählungen, Mädchen- und Familienbücher, psychologische und problemorientierte Werke, Romane mit der Abkehr von idealtypischen Figurenkonzepten und Handlungsmustern und mit der Enttabuisierung der bis dahin ausgesparten Bereiche wie Sucht, Alkohol- und Drogenkonsum, Inklusion, Flucht, Umgang mit Homosexualität, Gewalt, Erkrankungen und Tod sowie Mobbing. Die realistische Kinderund Jugendliteratur wird oft durch Elemente des Phantastischen bereichert oder ist auf Romantik rekurrierend fantastisch. Sie gewinnt an psychologischer Tiefe und Differenziertheit. Es kommt zu einer vielfältigen Symbiose traditioneller und moderner Formen, Inhalte, Motive und Funktionen sowie zu Adaption von Erzählstrukturen aus der allgemeinen Literatur. Die Distanz zur dieser verringert sich, verschwindet allmählich.

\section{WEGE ZUR LITERATUR IN LITERATUR}

Grundsätzlich kann die Kinder- und Jugendliteratur per se als eine lesefördernde Maßnahme mit einer steuernden Wirkung aufgefasst werden. Ihr Beitrag zu einer diskursiven Verhandlung dessen, was Literarität sein kann, wie sie gefördert werden kann, wie sie sich schließlich in Bildungskomponenten manifestiert, ist entscheidend.

Leseanimierend wirken Verhandlungen von Literalität in der Kinderund Jugendliteratur oder Literatur, die von dieser Gruppe gelesen wird bzw. gelesen werden muss, durch die literarisch-ästhetische, kulturpoetische Darstellung des Lesens. So trifft man auf Beschreibungen der tiefempfundenen Lesefreude in der Unendlichen Geschichte (Ende 1979) oder im Werk Die Stadt der träumenden Bücher (Moers 2004) oder bei Tolstoi (1980) in Detstvo, Otročestvo, Junost' (Kindheit, Knabenjahre, Jünglingszeit).

Ein anderer Weg führt über Einblicke in den sinnkonstruierenden Leseverstehensvorgang und in die damit verbundene Förderung eines positiven Selbstlesekonzepts als Leserin oder Leser (auch im Umgang mit Leseschwie- 
rigkeiten). Das beglückende Gefühl, das Gratifikationserlebnis, das die Protagonistinnen und Protagonisten nach der Bewältigung der Lesehürden empfinden, sollte ansteckend wirken (Dragunskij 1959; Zusak 2005; Zusak 2008).

Die Schriftstellerinnen und Schriftsteller können Leselust durch ein Labyrinth intertextueller Bezüge fördern, indem Titel genannt und / oder Namen von Figuren aus den (kanonisierten, klassischen) Werken in die eigenen Narrationen eingebunden werden. Dadurch wird eine subtile, sublime Verlockung zum Lesen wirksam erzielt (Aleksin 1976; Puškin 1833/1965; Kästner 1929/2010; Funke 2003). Bei Aleksin (1976) steigert sich der Lesegenuss des Hauptprotagonisten durch die von Eltern verbotene Lektüre - Anna Karenina von Tolstoj -, die ihm vor allem ein neues Selbstverständnis und eine andere Einstellung zur Welt verschafft. Die Bücher regen im Hauptprotagonisten ein tieferes Verständnis zwischenmenschlicher Beziehungen, Empathie und Imagination an. Funke (2003) bindet zahlreiche inter- und paratextuelle Elemente in die Narration ein und lässt sie aufgrund ihrer exponierten Positionierung sowie der Kursivsetzung leicht erkennen. Hinweise auf Michael Ende, Rudyard Kipling, John R. R. Tolkien, Paul Celan, Mark Twain, Lucy M. Boston erfüllen eine vorausweisende und rezeptionslenkende Funktion. Sie dienen der Charakterisierung der Figuren - unter anderem ihrer Belesenheit, die sie Außergewöhnliches erleben lässt. Generell ist die Intertextualität, vorausgesetzt man erkennt die Wiederaufnahmen zu literarischen Prä- oder Nachtexten und kann die intertextuellen Beziehungen für die Sinnkonstruktion des neuen Textes produktiv nutzen, ein äußerst effektives Mittel für die Steigerung der Lesefreude. Es lässt sich einem textanalytischen Spiel der Autoren von Intertexten mit literarischem Material, das sie vorfinden, gleichsetzten: erkennt eine Leserin oder ein Leser eine Allusion, kann er sich in die Rolle eines Beobachters begeben und daraus verfolgen, wie die künstlerische Idee vor dem Hintergrund der intertextuellen Aufnahme entwickelt wird. Kennt er die Regel nicht, wird er zum Entdecker, der sukzessiv das Rätsel löst und ggf. hierfür zunächst das Wissen aufbauen muss:

Für Leser wiederum kann das Entdecken von solchen Anspielungen zu einem lustvollen Spiel werden, bei dem er seine Wissenskompetenz auf die Probe stellt und im Falle des Erfolgs genießt (Anz 1998: 43).

Die Freude beim Erkennen und Entdecken der Intertextualität legt das Wahren des Spannungsverhältnisses zwischen Einfachheit und Komplexität nahe. Kinder- und Jugendbuchautorinnen und -autoren verfügen über andere Möglichkeiten sowohl bei der grundsätzlichen Herstellung von Fremdtextreferenzen als auch bei deren Markierung als Schriftstellerinnen und Schrift- 
steller, die sich an ein erfahreneres Lesepublikum richten. Explizite Markierungen, bspw. in Form von Erläuterungen, Kommentaren oder Fuß- bzw. Endnoten können den Lesefluss erschweren und dadurch die Lesefreude minimieren. Die impliziten intertextuellen Aufnahmen können wiederum unentdeckt bleiben, da anvisierte junge Rezipientinnen und Rezipienten noch nicht über eine intertextuelle Enzyklopädie verfügen (Broich \& Pfister 1985). Für sie sind folgende Aspekte von Bedeutung: Aktivieren von Wissensbeständen u.a. im Erkennen von Intertextualität; ein großer literarischer Erfahrungsschatz und der Aufbau des prozeduralen Wissens in Hinblick auf das Entdecken von intertextuellen Bezügen.

Der Weg zur Literatur kann über Figuren folgen, die täglich lesen, und dadurch Habitualisierung des Lesens bewirken (Moers 2004; 2017; Zusak 2005; 2008; Rowling 1998; Tolstoj 1980; Funke 2003). In C. Funkes Trilogie Tintenerz, Tintenblut, Tintentod (Funke 2003) entsteht durch das Lesen gute und schwarze Magie. Dieses Phänomen stellt eine Allusion an eine besondere Lesegratifikation dar, nämlich an das Eintauchen in phantastische Welten und somit an die Transzendenz der Realität. Bei Funke wird dieser Übergang durch das Überschreiten der Grenze zwischen erster und zweiter Diegese, also durch die Berührungspunkte der primären fiktiv-realistischen Alltagswelt und der sekundären fanstatischen Anderswelt (Nikolajeva 1988) sowie durch den Weltenwechsel der Figuren verstärkt. Die Weltenüberschreitung ergibt sich im Zwei-Welten-Konstrukt der Trilogie durch die Präsenz des Zauberhaften. Dem Lesenden - bei Funke explizit Mortimer Folchart, seiner Tochter Meggie, ihrer Tante Elinor, implizit jedem, der liest öffnen sich durch den (zauberhaften) Gebrauch des Buches phantastische Welten, die er betreten und verlassen kann. Mortimer liest in das intradiegetische Buch (Genette 1994) Tintenherz seine Frau Resa hinein und einen Bösewichten Capricorn heraus. Anschließend versucht er die narrative Metalepse (Genette 1994) umzukehren. Es handelt sich dabei um ein, zu spannenden und gefährlichen Abenteuern, führendes Unterfangen.

Ferner kann das Schaffen von Möglichkeiten zur Empathie für die Figuren zum Figurenverstehen führen. Grundsätzlich können sie als ein Wegbereiter für das Literaturverständnis fugieren: In der literaturwissenschaftlichen Forschung wird das Figurenverstehen als eine komplexe Konstruktion aufgefasst. Sie verbindet die textliche Figurengestaltung in der Figurenwahrnehmung mit sozio-emotionalem, kulturellem und literarischem Vorwissen in einem mentalen Figurenmodell, das kategorisiert, personalisiert oder individualisiert sein kann und eine rück- und vorausverweisende antizipierende Komponente umfasst (Eder 2008; Hartner 2012; Schneider 2000). Figuren können sich im Roman von C. Funke ihr Leben ohne Bücher nicht vorstellen: Meggies Tante Elinor ist eine leidenschaftliche Buchsammlerin, 
Meggies Vater Mortimer Folchart ist ein Buchrestaurator und dazu ein zauberisch-leidenschaftlicher Vorleser und Meggie selbst trennt sich niemals von Büchern - sogar nachts hat sie ein Buch unter ihrem Kopfkissen.

Die literarische Kommentierung von Literalität kann allegorisch-lehrhaft, wie in Fabeln, erfolgen, parabelhafte Erzählstrukturen aufweisen, historischkulturell aufgegriffen werden, bspw. als versperrte Zugänge zur Bildung aufgrund des natürlichen Geschlechts (Alekseeva 1985; Hahn 2002) oder einer niederen Schichtzugehörigkeit (Fonvizin 1953). Literalität kann als ein soziales Moment präsentiert werden, dennoch keine Garantie für den sozialen Aufstieg bedeuten. Sie kann als Mimikry und dadurch eine Chance für das Überleben (Belych \& Pantelejew 1927; Zusak 2005; 2008) oder Restitution des Lebens dargestellt sein (Auster 1989) oder als Substantialität fungieren (Ulickaja 2011).

Die folgenden Auszüge aus kinder- und jugendliterarischen Werken können in aller Exemplarität als Beispiele für aufgezeigte Wege zur Literalität fungieren.

Beispiel 1: Дядя Фёдор, пес и кот / Djadja Fedor, pes i kot / Onkel Fjodor der Hund und der Kater von Uspenskij (1974: 3)

- А кто тебя разговаривать научил? - спрашивает дядя Фёдор.

- Да так, - говорит кот.- Где слово запомнишь, где два. А потом, я у профессора одного жил, который язык зверей изучал. Вот и выучился. Сейчас без языка нельзя. Пропадёшь сразу, или из тебя шапку сделают, или воротник, или просто коврик для ног (Uspenskij 1974: 3).

- Wer hat dir die Sprache beigebracht? - fragt Onkel Fjodor.

- Mal hier mal da schnappt man ein Wort auf und es bleibt im Gedächtnis hängen, und dann lebte ich eine Zeit lang bei einem Professor, der sich für Sprache der Tiere interessierte, so lernte er meine und ich seine Sprache. Heutzutage geht man ja ohne Sprachkenntnisse unter, oder man wird zur Mütze verarbeitet oder gar noch schlimmer, endet wie ein Fußabtreter (dt. Übersetzung der Verfasserin Uspenskij 1974: 3).

Sprechende Tiere sind in den kinderliterarischen Werken ein oft anzutreffendes Element. Besonders ist die Rolle, die der Kater der Sprache und der Beherrschung kommunikativer Kompetenz in Wort und Schrift zuschreibt. Ohne die Fähigkeit, sich den Anderen mitzuteilen und kommunikativ auf ihre Bedürfnisse einzugehen, schätzt er die Überlebenschancen in der gegenwärtigen Gesellschaft als gering bzw. als nicht existent ein. Der eloquente Matroskin erzielt dank der Sprachkenntnisse, der kommunikativen und der Verstehenskompetenz eine wesentliche und dauerhafte Verbesserung seiner Lebenssituation. Er wird nicht nur zu Onkel Fjodor nach Hause eingeladen, wo er mit Leckerbissen verwöhnt wird, sondern zieht in ein eigenes 
Haus ein (Uspenskij 1974). In der medialen Umsetzung von Popov (1975) diktiert der Kater zunächst einen Brief und im späteren Verlauf der Narration schreibt er sogar einige Abschnitte des Briefes an die Eltern selbst und hält sich sowohl an die schriftsprachlichen Konventionen als auch an die geltenden Normen der Briefkommunikation im privaten Umfeld. Auch der Hund Šarik beherrscht Sprache in Wort und Schrift, abonniert eine Zeitschrift und stellt sich als ein begeisterter Leser heraus. Neben der bestellten Fachzeitschrift werden Zeitungen gelesen und es finden regelmäßige Berichte, im Sinne der Anschlusskommunikation, über das Gelesene statt. Die kleine Dohle bekommt die Sprache vom Kater beigebracht und setzt sie adäquat, in der Rolle des Wachhundes, ein.

Versucht man eine Hierarchie zwischen Menschen und Tieren auf die sozialen Gruppen einer Gesellschaft zu übertragen, stellt man fest, dass Literalität nicht an einen sozialen und kulturellen Sozialisierungshintergrund gebunden, sondern, ganz im Gegenteil für alle als eminent dargestellt wird. Man denke an die wissenschaftliche Bearbeitung der Dependenz des restringierten und elaborierten Codes und der Schichtzugehörigkeit von Bernstein 1971. Diese gilt zwar nicht als konsensfähig, aber sie inspirierte Untersuchungen $\mathrm{zu}$ basic interpersonal communicative skills (BICS) und cognitive academic language proficiency (CALP) (Cummins 1979) und ihre nahezu inflationäre Verbreitung in gegenwärtigen Fremd- und Zweitspracherwerbskonzepten.

Beispiel 2: Приключения Незнайки и его друзей / Prikljuс̌еnija Neznajki i ego druzej / Abenteuer von Neznaika und seiner Freunde von Nosov (1953/1992: 6)

В одном домике на улице Колокольчиков жило шестнадцать малышейкоротышей. Самым главным из них был малыш-коротыш, по имени Знайка. Его прозвали Знайкой за то, что он знал очень много. А знал он много потому, что читал разные книги. Эти книги лежали у него и на столе, и под столом, и на кровати, и под кроватью. В его комнате не было такого места, где бы не лежали книги. От чтения книг Знайка сделался очень умным. Поэтому все его слушались и очень любили (Nosov 1992: 6).

In einem Häuschen auf der Straße der Glöckchen lebten sechzehn Knirpse. Ihr Anführer hieß Znaika (Immerklug). Man nannte ihn so, weil er sehr, sehr viel wusste. Er wusste viel, weil er viele unterschiedliche Bücher las. Sie lagen bei ihm auf dem Tisch und unter dem Tisch, auf dem Bett und unter dem Bett. In seinem Zimmer gab es nirgendwo einen Platz, wo keine Bücher lagen. Vom Lesen wurde er sehr, sehr klug. Deswegen horchten alle auf sein Wort und liebten ihn (dt. Übersetzung der Verfasserin Nosov 1992: 6).

Man hat in der Passage aus dem kinderliterarischen Werk von Nosov sowohl diskursiv als auch mental mit einem wirkungsmächtigen Modell zu 
tun: Jemand der viel liest, liest gut, und jemand der gut und viel liest, weiß viel, jemand der viel weiß, wird geliebt. Darüber hinaus nimmt er die Rolle des Leaders ein. Znaika ist belesen und stets bestrebt, sein Wissen zu erweitern, und dieses zum Nutzen der Gesellschaft einzusetzen. Einerseits wird somit auf das Nützlichkeitsprinzip der erworbenen Literarität und der kontinuierlichen Vervollkommnung der Kenntnisse hingewiesen, andererseits veranschaulicht der Auszug, wie ein breitgefächertes und ausdifferenziertes Wissen, das auf dem individuellen Bildungsweg durch das Lesen erworben wird, bestimmte Lebenswege ebnet, indem sie andere ausschließt. Bücher jeden Inhalts und ihre Vielzahl sind in dem, in diesem kinderliterarischen Werk, anvisierten Bildungskonzept bedeutsam.

Der Zusammenhang von Menge und Können ist vielen Förderprogrammen weltweit inhärent. Die Lesemenge wird als ein entscheidender Faktor betrachtet und so sollen die Kinder und später Jugendliche sowohl in der Freizeit als auch im schulischen Literaturunterricht möglichst viele Autoren kennenlernen und vertieft behandeln. Die Listen mit Lektüreempfehlungen, in denen die schulische Bildung legitimiert wird, sind als Zeugnis anzuführen (Karg \& Kuzminykh 2014). Es kann allerdings sein, dass das Viellesen nicht zwangsläufig zum Verstehen und zur Literarität führt. Gehen die Schwierigkeiten nicht mit einer abstinenten Lesehaltung und / oder einer geringen Motivation einher, sondern ergeben sich aus kognitiven Dispositionen, hat die Quantität der Lektüre kaum positive Effekte auf den verstehenden Lesevorgang - "deep reading“ (Nikolajeva 2014: 14). Das ist der Fall, wenn die Rezipientinnen und Rezipienten über die Ebene der Oberflächenrepräsentation nicht zu den hierarchiehöheren Ebenen des Verständnisses gelangen können. D. h. keine Inferenzen auf der propositionalen Ebene, der Ebene des mentalen Modelles, der Genre- und Kommunikationsebene vornehmen können (Kuzminykh 2018). In einem kinderliterarischen Werk können nicht die kognitionspsychologischen Grundlagen im Einzelnen aufgegriffen werden, aber es kann der Umgang mit Schwierigkeiten und der Prozess der sukzessiven Überwindung der Leseherausforderungen durch das positive Selbstkonzept als Leserin oder Leser, durch den Aufbau vom ausdifferenzierten Wissen und durch die Erweiterung des kulturellen, sprachlichen und literarischen Horizonts als eine Art babylonische Mannigfaltigkeit und eine gewisse Hartnäckigkeit demonstriert werden. Die Auszüge aus den Werken Bücherdiebin (Zusak 2008) und Deniskiny rasskazy (Dragunskij 1959) können als Kern des Leseerwerbdiskurses betrachtet werden.

Beispiel 3: Die Bücherdiebin von Zusak (2005: 66, 67, 69) und Zusak (2008: 72ff.).

You wouldn't think it, she wrote, but it was not so much the school who helped me to read. It was Papa. People think he's not so smart, and it's true that he do- 
esn't read too fast, but I would soon learn that words and writing actually saved his live once (Zusak 2005: 66).

[...] but she was going to read. She was going to read the book. The excitement stood up in her. Visions of a ten-year-old-reading genius were set alight. If only it was that easy. "To tell you the truth", Papa explained up front, "I am not such a good reader myself". But it didn't matter that he read slowly. If anything, it might have helped that his own reading pace was slower than average. Perhaps it would cause less frustration in coping with the girl's lack of ability (Zusak 2005: 67).

Looking back, Liesel could tell exactly what her papa was thinking when he scanned the first page of The Gravedigger's Handbook. As he realised the difficulty of the text, he was clearly aware that such a book was hardly ideal. There were words in there that he'd have trouble with himself. Not to mention the morbidity of the subject (Zusak 2005: 69).

When he made her point out any words she could read and actually say them, there were only three - the three main German words for 'the'. The whole page must have two hundred words on it. That might be harder than I thought. She caught him thinking it, just for a moment (Zusak 2005: 69).

Man kann es kaum glauben, schrieb sie, aber in der Schule habe ich das Lesen nicht gelernt. Papa hat es mir beigebracht. Die Leute denken, dass er nicht besonders klug ist, und es stimmt, dass er nicht besonders schnell lesen kann, aber ich sollte bald erfahren, dass Worte und das Schreiben ihm einmal das Leben gerettet haben (Zusak 2008: 72).

[...], aber sie würde lesen lernen. Sie würde das Buch lesen. Die Erregung stand ihr ins Gesicht geschrieben. Sie sah sich bereits als ein zehnjähriges Wortewunder. Wenn es bloß so einfach wäre. „Um die Wahrheit zu sagen“, erklärte Papa rundheraus, „ich kann selbst nicht besonders gut lesen.“ Aber es machte nichts, dass er langsam las. Im Gegenteil, es war womöglich hilfreich, dass sein Lesetempo unter dem Durchschnitt lag. Vielleicht half es, die Ungeduld und die Enttäuschung angesichts von Liesels Unvermögen abzumildern (Zusak 2008: 73). [...]

Rückblickend wusste Liesel ganz genau, was ihr Papa dachte, als er das Handbuch für die Totengräber durchblätterte. Er erkannte, wie schwierig der Text war und wie wenig er für ihr Vorhaben geeignet war. Da standen Worte, mit denen sogar er Probleme hatte. Ganz zu schweigen von dem düsteren Thema des Buchs (Zusak 2008: 74).

Es waren nur drei - ,der', ,die' und ,das'. Auf der ganzen Seite standen insgesamt etwa zweihundert Worte. Das wird schwieriger als ich dachte. Sie erwischte ihn dabei, wie er das dachte (Zusak 2008: 75).

Dem Lesenlernen und der Literatur kommt im Roman eine lebenskonstituierende und eine lebensrettende Funktion zu (Kuzminykh 2018). Liesel erliest 
mühsam die Wörter: Sie versucht den Propositionen Sinn zu geben, stellt sich mentale Bilder vor, konstruiert Situationsmodelle des Gelesenen und das an einem Thema (Beerdigung), das einem Kind fremder nicht sein kann. Schließlich wagt sie Wörter in ihrer ästhetischen Funktion und Wirkung zunächst aufgemuntert von Max, später um ihn aufzumuntern, zu gebrauchen (Zusak 2005: 268; 2008: 272). Ihrer Unfähigkeit zu lesen, wird mit Intoleranz seitens der neuen Mitschülerinnen und Mitschüler begegnet. Dennoch gibt sie nicht auf. Lesen ist in erster Linie durch konzentrierte Aufmerksamkeit und mühsames Erlernen der Lesetechnik bestimmt, aber Liesel meistert diese Aufgabe und wird zu Schriftstellerin.

Beispiel 4: Deniskiny rasskazy von Dragunskij 1959.

Einmal kaufe sich Miška ein Buch mit dem Titel „Trigonometrische Funktionen und Polynomen“ von Čebyšev. Klar, hat er kein einziges Wort in diesem Buch verstanden und beschloss, das Buch später zu lesen, wenn er klüger wird. Seitdem steht das Buch im Regal und wartet darauf, dass Miška klüger wird.

Eine solche positive Rückmeldung im Hinblick auf das Resultat der Lektüre eines Sachbuchs trägt zur Förderung eines positiven Selbstkonzepts als Leser bei und kann eine beruhigende Wirkung auf die jungen Leser haben. Das Nicht-Verstehen wird auf die noch nicht vorhandenen Wissensbestände zurückgeführt. Später, wenn sich Miška mathematisches Wissen, Vorstellungen von Funktionen und Polynomen und Trigonometrie angeeignet haben wird - also klüger geworden ist -, wird er das Buch verstehen können. Diese Vorstellung korreliert mit den kognitionspsychologischen Modellen des Lesens und mit der hermeneutischen Tradition, in der es vom Vorurteil in Sinne des Vorwissens ausgegangen wird (Gadamer 1990).

Zum positiven Selbstkonzept (als Leser) trägt das Lesen in der Trilogie von A. Steinhöfel (2008) bei. Der gutherzige, naive und sich durch eine erhöhte Emotionalität und Begeisterungsfreude von anderen Kindern unterscheidende Rico verkörpert den kinderliterarischen Archetyp der Romantik. Er begegnet der Welt intuitiv und unerschrocken. Der hochbegabte, rationalvernünftige und vorsichtige, die Wirklichkeit reflektierende Oscar entspricht dem Aufklärungsideal. Beide Figuren lesen - Rico, um „schlauer zu werden" (Steinhöfel 2008: 18), Oscar aus demselben Grund und aufgrund der Zufluchtsmöglichkeit, die ihn vor Einsamkeit und Ausgrenzung rettet.

\section{Beispiel 5: Rico, Oscar und Tieferschatten von Steinhöfel (2008)}

Ich gucke immer im Lexikon nach, was ich nicht kenne, um schlauer zu werden. Manchmal frage ich auch, Mama oder Frau Dahling oder meinen Lehrer, den Wehmeyer. Was ich rausgefunden habe, schreibe ich dann auf (Steinhöfel 2008: 15). 
In dem Auszug von Tolstoj (1980) wird der Rezipient mit dem berückenden Eintauchen des Protagonisten in die fiktionale Welt der abwechslungsreichen Bilder, Begebenheiten und fremdartigen, kuriosen Eindrücken konfrontiert. Nikolenka ist von den französischen Romanen und ihrer obsessiven Anziehungskraft überwältigt. Er erfreut sich an den Phantasien und Helden sowie am Leseerlebnis selbst.

Beispiel 6: Детсbо, отрочестbо, юность / Detstvo, Otročestvo, Junost' / Kindheit, Knabenjahre, Jünglingszeit von Tolstoj (1980: 279)

Чтение французских романов, которых много привез с собой Володя, было другим моим занятием в это лето. В то время только начинали появляться Монтекристы и разные „Тайны“, и я зачитывался романами Сю, Дюма и Поль де Кока. Все самые неестественные лица и события были для меня так же живы, как действительность, я не только не смел заподозрить автора во лжи, но сам автор не существовал для меня, а сами собой являлись передо мной, из печатной книги, живые, действительные люди и события. Ежели я нигде не встречал лиц, похожих на те, про которых я читал, то я ни секунды не сомневался в том, что они будут. Я находил в себе все описываемые страсти и сходство со всеми характерами, и с героями, и с злодеями каждого романа, как мнительный человек находит в себе признаки всех возможных болезней, читая медицинскую книгу. Нравились мне в этих романах и хитрые мысли, и пылкие чувства, и волшебные события, и цельные характеры (Tolstoj 1980: 279).

Eine andere Leidenschaft, die mich diesen Sommer packte, war das Lesen französischer Romane. Zu diesem Zeitpunkt begannen erst die Romane über Monte Christo und "Geheimnisse“ zu erscheinen. Mit Begeisterung las ich die Romane von Su, Dumas, und Paul de Cock. Alle, sogar die unnatürlichsten Protagonisten und Ereignisse erschienen mir lebendig und real. Ich dachte nicht daran, dass der Autor alles ausgedacht hat. Für mich existierte der Autor nicht. Alle im Buch beschriebenen Details standen vor meinem Auge. Wenn auch ich noch nie in den Romanen lebenden Personen begegnete, zweifelte ich keine Sekunde lang, dass es noch kommt und in der nahen Zukunft geschieht. Ich suchte in mir nach den darin genannten Eigenschaften und nach Ähnlichkeiten mit Protagonisten, wie wenn ein leicht zu beeinflussender Mensch der bei der Lektüre eines medizinischen Buches alle möglichen Krankheiten bei sich feststellt. Ich durchlebte fantastische Ereignisse, in meinem Herzen entflammten starke Gefühle, in meinem Kopf entstanden listige Gedanken (dt. Übersetzung der Verfasserin Tolstoj 1980: 279).

Die evasorische Lektüre entbindet Nikolenka von Wirklichkeit und lässt ihn Figuren und Erlebnisse aus fiktiven (sekundären) Welten in die reale (primäre) metaleptisch übertragen. Er liest identifikatorisch und empathisch, nicht reflektierend, kritisch oder distanzierend. Für ihn sind Figuren in Ro- 
manen ein Faszinosum. Darüber hinaus lernt der lesende junge Mann aus der Lektüre fürs Leben:

Сколько я с помощью романов придумал различных французских фраз для Колпикова, ежели бы я когда-нибудь с ним встретился, и для нее, когда я ее, наконец, встречу и буду открываться ей в любви! Я приготовил им сказать Такое, что они погибли бы, услышав меня (Tolstoj 1980: 279).

Wie viele neue französische Phrasen dachte ich mir mit Hilfe der französischen Romane für Kolpikov aus, wenn ich ihm eines Tages begegnete, und für sie, wenn das Schicksal uns endlich zusammenführt, und ich ihr meine Liebe gestehe. Ich war bereit ihnen Solche Dinge (Hervorhebung um Original) zu sagen, dass wenn sie mich hörten, sie sofort sterben würden (dt. Übersetzung der Verfasserin Tolstoj 1980: 279).

На основании романов у меня даже составились новые идеалы нравственных достоинств, которых я желал достигнуть. Прежде всего я желал быть во всех своих делах и поступках „noble“ (я говорю noble, а не благородный, потому что французское слово имеет другое значение, что поняли немцы, приняв слово nobel и не смешивая с ним понятия ehrlich [*]), потом быть страстным и, наконец, к чему у меня и прежде была наклонность, быть как можно более comme il faut. Я даже наружностью и привычками старался быть похожим на героев, имевших какое-нибудь из этих достоинств (Tolstoj 1980: 279).

Auf der Grundlage von den gelesenen Romanen bildeten sich bei mir neue Ideale, die ich erreichen wollte, heraus. Vor allem wollte ich in allen meinen Taten "noble“ sein (ich verwende das Wort noble und nicht edel, weil die französische Bezeichnung, eine andere Bedeutung hat, was die Deutschen begriffen und daher nobel entlehnt haben, ohne dieses Wort mit dem Begriff ehrlich zu vermischen), dann wollte ich leidenschaftlich sein, und dann, zu dem, was ich schon früher eine Neigung hatte, so wie es nur möglich ist comme il faut. Ich wollte sogar mit meinem Äußeren und mit meinen Gewohnheiten den Helden ähneln, die eine von diesen Eigenschaften hatten (dt. Übersetzung der Verfasserin Tolstoj 1980: 279).

Auffallend in dieser Passage ist die Verwendung von Fremdwörtern bei der Figurenbeschreibung, die auf die sprachlich-ästhetische Verfasstheit des Textes Aufmerksamkeit lenken: Mit noble und comme il faut wird nicht einfach die Unübersetzbarkeit dieser Lexeme, sondern das Vorhandensein von russischen Äquivalenten für dieselben Denotate negiert. Beide Wörter lassen sich durchaus in die russische Sprache übertragen - comme il faut als прилично, благородно - angemessen; noble als благородный - edel. Vielmehr werden mit dieser Unübersetzbarkeit die französischen Elemente im begrifflichen Inhalt der sprachlichen Zeichen statuiert - also mit rein russischen 
Konstituenten werden weder noble noch comme il faut zu erreichen sein. D.h., es gibt in der russischen Kultur keine Realien und keine entsprechenden Bezeichnungen für sie. Zum Nachdenken regt die Tatsache an, dass auch in der deutschen Kultur, wie vom Protagonisten angemerkt wird, kein entsprechendes Äquivalent für noble geben soll. Ehrlich deckt den semantischen Raum von noble nicht ab, dies legitimiert die Verwendung des Fremdwortes in der deutschen Sprache. Ebenso verhält es sich mit comme il faut, auch wenn darauf im Text nicht eingegangen wird. Es kann aber auch als ein Kunstgriff gedeutet werden, die Redundanz der ins Russische übertragenden lexikalischen Einheiten zu vermeiden. Aus der historischen Perspektive betrachtet, trifft man in dieser Passage auf das Phänomen des kulturellen Dialogs und auf eine partielle Codeüberlagerung. Man kann darin sogar eine Art Abwertung der russischen und auch der deutschen Kultur und die Aufwertung der französischen sehen, oder aber eine Kritik an den sprachpuristischen Tendenzen des 19. Jahrhunderts in beiden Ländern. Eine vergleichbare Verwendung von französischen Lexemen, die sich als ein intertextueller Hinweis auffassen lässt, findet sich bei der Charakterisierung von Tatjana in Evgenij Onegin (Puškin 1965: 148). Sie wird als ein authentisches Abbild / eine treue Kopie von comme il faut beschrieben. Darauf folgt die Bitte um Entschuldigung, die an Šiškov, den Leiter des Akademie-Wörterbuchs, der ein strenges sprachpuristisches Konzept verfolgte, gerichtet ist. Dies legt nahe, dass Puškin die angebliche Nichtübersetzbarkeit von comme il faut satirisch, als eine Spitze, gegen den Sprachpurismus einsetzte. Gegen die Abwertung der russischen Kultur spricht darüber hinaus die Verwendung des Anglizismus vulgar bei der Charakterisierung der Hauptprotagonistin (Puškin 1965: 149), die in der darauffolgenden Strophe folgt. Diese Eigenschaft ist ebenfalls mit den rein russischen Mitteln nicht zu erreichen. Somit stellt Puškin das Gleichgewicht her, worauf Tolstoj in der zitierten Passage verzichtet.

Vom Interesse ist die Erwähnung von kanonisierten literarischen Werken, die Aufmerksamkeit der Leserinnen und Leser auf diese Texte und Autoren lenken. Diese können einerseits als kulturhistorische Aussagen über Lesesozialisation ganzer Generationen und kulturelle Identität von bestimmten gesellschaftlichen Schichten wahrgenommen werden. Andererseits wecken sie die Leseneugier der Rezipientinnen und Rezipienten. Die Komik mit der die Passage abgeschlossen wird, potenziert die Wissbegierde und das Lesevergnügen:

Помню, что в одном из прочитанных мною в то лето сотни романов был один чрезвычайно страстный герой с густыми бровями, мне так захотелось быть похожим на него наружностью (морально я чувствовал себя точно 
таким, как он), что я, рассматривая свои брови перед зеркалом, вздумал простричь их слегка, чтоб они выросли гуще, но раз, начав стричь, случилось так, что я выстриг в одном месте больше, - надо было подравнивать, и кончилось тем, что я, к ужасу своему, увидел себя в зеркало безбровым и вследствие этого очень некрасивым (Tolstoj 1980: 279).

Ich erinnere mich, dass es sich in einem Roman - ich diesem Sommer las ich Hunderte davon - um einen besonders leidenschaftlichen Helden handelte. Er hatte prächtige Augenbrauen. Ich verspürte ein unstillbares Verlangen, ihm äußerlich ähnlich zu sein (innerlich war die Übereinstimmung zwischen ihm und mir vollkommen). Ich betrachtete lange mein Spiegelbild und beschloss die Augenbrauen leicht zu stützen, damit die wuschiger wachsen. Aber einst angefangen, passierte es, dass ich an einer Stelle mehr herausschnitt als zunächst beabsichtigt, - ich bemühte mich um die Korrektur, und schließlich endete das Ganze damit, wie ich mit Schrecken feststellte, dass ich ganz ohne Augenbrauen war, und folglich sehr unschön aussah (dt. Übersetzung der Verfasserin Tolstoj 1980: 279).

\section{FAZIT}

Die stärksten, die schönsten und die bestimmenden Leseerlebnisse sind die der Kindheit und Jugend. Es ist der Zauber der Kinder- und Jugendliteratur, der Menschen zu Lesern macht. Die Hinführung zur Literalität erfolgt auf unterschiedlichen Wegen. Es wird die Wertschätzung des Lesen- und Schreibenkönnens demonstriert. Zu weiteren Strategien gehören die Darstellungen des beflügelnden Gefühls und der Gratifikationserlebnisse, die sich nach der Überwindung von potenziellen Leseschwierigkeiten einstellen. Das führt zur Herausbildung eines positiven Selbstlesekonzepts. Es folgen sublime und gerade deswegen effektive Anregungen zur Erweiterung des literarischen Horizonts - ein Labyrinth intertextueller Bezüge -, Anregungen zum Erwerb der sprachlich-literarischen Fähigkeiten und Fertigkeiten und schließlich des literarischen Wissens. Es werden Identifikationsmöglichkeiten mit lesenden Figuren geschaffen und das Eintauchen in die fantastischen Welten ermöglicht. Kinder- und jugendliterarische Werke können Wegbereiter zur literarästhetischen Komplexität sein sowie Sinn und Verständnis dafür schaffen, was eine Kulturgemeinschaft als ihre Literatur entwickelt hat.

\section{LITERATURVERZEICHNIS}

Alekseeva, A. (1985). Kolokol'čik. Moskva: Detskaja literatura. Aleksin, A. (1976). Pozdnij rebenok. Moskva: Detskaja literatura. Anz, T. (1998). Literatur und Lust. München: dtv. 
Arnim, A. / Brentano C. (1808). Des Knaben Wunderhorn. Heidelberg: Mohr \& Zimmer.

Auster, P. (1989). Moon palace. New York: Viking Press.

Bachtin, M. (1979). Slovo v Romane. In: R. Grübel (Hrsg.), Die Ästhetik des Wortes (S. 219-251). Frankfurt a. M.: Suhrkamp.

Belych, G. / Panteleev, L. (1927). Respublika ŠKID. Moskva: Škol'naja bibliotheka.

Bernstein, B. (1971). Class, codes and control. Theoretical studies towards a sociology of language. London: Routledge.

Broich, U. / Pfister, M. (1985). Intertextualität. Formen, Funktionen, anglistische Fallstudien. Tübingen: Niemeyer.

Bürger, A. (1786/1976). Wunderbare Reisen zu Wasser und Lande, Feldzüge und lustige Abenteuer des Freiherrn von Münchhausen, wie er dieselben bei der Flasche im Zirkel seiner Freunde selbst zu erzählen pflegt. Frankfurt a. M.: Insel.

Busch, W. (1865). Max und Moritz. Eine Bubengeschichte in sieben Streichen. München: Braun und Schneider.

Campe, H. (1779). Robinson der Jüngere zur angenehmen Unterhaltung der Kinder. Hamburg: Bohn.

Carroll, L. (1865). Alice in Wonderland. London: Macmillan \& Co.

Cummins, J. (1979). The Influence of bilingualism on cognitive growth: A synthesis of research findings and explanatory hypotheses. Working Papers on Bilingualism, 9, 2-44.

Defoe, D. (1719). The life and strange, surprising adventurens of Robinson Croesoe, of York, Mariner: Who lived eight and twenty years, all alone in an un-inhabited island on the coast of AMERICA, near the Mouth of the Great River of OROONOQUE; having been cast on shore by shipwreck, wherein all the men perished but himself. WITH an account how he was at last as strangely deliver'd by PYRATES. Written by himself. London: Taylor.

Dragunskij, V. (1959). Deniskiny rasskazy. Moskva: Detskaja literatura.

Eder, J. (2008). Die Figur im Film. Grundlagen der Figurenanalyse. Marburg: Schüren.

Ende, M. (1979). Die unendliche Geschichte. Stuttgart: Thienemann.

Ewers, H.-H. (2012). Literatur für Kinder und Jugendliche. Eine Einführung. München: Fink.

Fonvizin, D. (1953). Brigadir. Moskva: Detskaja literatura.

Funke, C. (2003). Tintenwelt: Tintenherz. Hamburg: Dressler.

Funke, C. (2005). Tintenwelt: Tintenwelt. Hamburg: Dressler.

Funke, C. (2007). Tintenwelt: Tintentod. Hamburg: Dressler.

Gadamer, H.-G. (1990). Hermeneutik I. Wahrheit und Methode. Grundzüge einer philosophischen Hermeneutik. Tübingen: Mohr.

Genette, G. (1994). Die Erzählung. München: Fink.

Grimm, J. / Grimm, W. (1962). Kinder- und Hausmärchen. Gesammelt durch die Brüder Grimm. 2 Bände. Berlin 1812 u. 1815. Durchgesehen und der heutigen Schreibart angeglichen. Frankfurt a. M.: Fischer.

Hahn, U. (2002). Das verborgene Wort. Stuttgart: DVA.

Hartner, M. (2012). Perspektivische Interaktion im Roman. Kognition, Rezeption, Interpretation. Berlin: De Gruyter.

Hoffmann, H. (1844/1917). Der Struwwelpeter oder lustige Geschichten und drollige Bilder für Kinder von 3-6 Jahren. Frankfurt a. M.: Literarische Anstalt Rütten \& Loening.

Iser, W. (1970). Die Appellstruktur der Texte. Konstanz: UTB.

Karg, I. / Kuzminykh, K. (2014). Sprache und Literatur als Bildungskomponenten. Diskurs, Historie und Empirie. Frankfurt a. M.: Peter Lang.

Kästner, E. (1976). Pünktchen und Anton. Hamburg: Dressler.

Kästner, E. (2010). Emil und die Detektive. Hamburg: Dressler. 
Key, E. (1978). Das Jahrhundert des Kindes. Königstein i. Ts.: Athenäum-Verlag.

Kintsch, W. (1998). Comprehension. A paradigm for cognition. Cambridge: CUP.

Kuzminykh, K. (2018). Jugendliterarische Werke im interkulturellen, medienintegrativen Litertaturunterricht. Glottodidactica. An International Journal of Applied Linguistics, 45, 85-104.

Lindgren, A. (1949). Pippi Langstrumpf. Hamburg: Oettinger.

Lukian (2014). Die Hauptwerke des Lukian. Berlin: De Gruyter.

Mitterer, N. / Wintersteiner, W. (2015). Literarische Erfahrung. Ästhetischer Modus und literarisches Lernen. Leseräume, 2, 96-108.

Moers, W. (2004). Die Stadt der träumenden Bücher. München: Pieper.

Moers, W. (2017). Prinzessin Insomnia und der alptraumfarbene Nachtmar. München: Knaus.

Nikolajeva, M. (1988). The magic code: The use of magical patterns in fantasy for children. Stockholm: Almqvist \& Wiksell International.

Nosov, N. (1992). Priključenija Neznajki i ego druzej. Čeljabinsk: Plastik-Inform.

Novalis (1977). Schriften. Die Werke Friedrich von Hardenbergs. In: P. Kluckhohn (Hrsg.), Das dichterische Werk. Darmstadt: Wissenschaftlichen Buchgesellschaft.

Paul, J. (1963). Levana oder Erziehungslehre. Paderborn: Schöningh.

Popov, V. (1975): Troje iz Prostokvašino. Filmreihe. Moskva: Sojuzmultfil'm.

Puškin, A. (1965). Evgenij Onegin. Moskva: Detskaja literatura.

Rowling, J. (1998). Harry Potter und der Stein der Weisen. Hamburg: Carlsen.

Reuter, C. (1696). Schelmuffskys kuriose und sehr gefährliche Reisebeschreibung zu Wasser und Land. Kassel: [o. V.].

Schneider, R. (2000). Grundriß zur kognitiven Theorie der Figurenrezeption am Beispiel des viktorianischen Romans. Tübingen: Stauffenberg-Verlag.

Seemann, C. (1898). Die Struwwel-Lene oder Böse Streiche eines ungezogenen Mädchens. Dresden: Liebes \& Teichner.

Steinhöfel, A. (2008). Rico, Oscar und Tieferschatten. Hamburg: Carlsen.

Stevenson, R. L. (1988). A Gossip of Romance. In: R. L. Stevenson (Hrsg.), The lantern-bearers and other essay (S. 172-182). London: Chatto \& Windus.

Stockwell, P. (2002). Cognitive poetics. An introduction. London: Routledge.

Storm, T. (1877). Theodor Storm's gesammelte Schriften. Band 9. Braunschweig: Westermann.

Tolstoj, L. (1980). Detstvo, oročestvo, junost'. Leningrad: Chudožestvennaja literatura.

Ulickaja, L. (2011). Zelenyj Šater. Moskva: Eksmo.

Uspenskij, E. (1974). Djadja Fëdor, Pës i Kot. Moskva: Detskaja literatura.

Weiße, C. F. (1758). Scherzhafte Lieder. Leipzig: Weidemannischen Buchhandlung.

Wieler, P. (2003). Varianten des Literacy-Konzepts und ihre Bedeutung für die Deutschdidaktik. In: A. Ulf (Hrsg.), Deutschdidaktik und Deutschunterricht nach PISA (S. 47-68). Freiburg: Fillibach.

Worthmann, F. (2004). Literarische Wertungen. Vorschläge für ein deskriptives Modell. Wiesbaden: DUV.

Wrobel, D. (2010). Kinder- und Jugendliteratur nach 2000. Praxis Deutsch, 224, 4-11.

Zusak, M. (2008). Die Bücherdiebin. München: Blanvalet / Random House.

Zusak, M. (2005). The book thief. Sydney: Picador.

Received: 9.01.2019; revised: 3.08.2019 